\title{
Evaluation of carnitine levels in dried blood spot samples in children with autism spectrum disorder
}

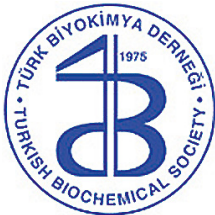

https://doi.org/10.1515/tjb-2020-0420

Received August 26, 2020; accepted December 4, 2020;

published online September 6, 2021

\section{Abstract}

Objectives: Autism Spectrum Disorder is a neurodevelopmental disease with an average diagnosis age of over 3 years. Carnitine levels in ASD are important because they show potential mitochondrial dysfunction and abnormal fatty acid metabolism. In this study, in ASD children carnitine levels in dried blood spot samples were evaluated and compared with the control group.

Methods: Twentythree children diagnosed with ASD in Research and Training Hospital (19 boys, 4 girls) and age and gender matched 24 children without ASD were enrolled in this study. 17 carnitines in dried blood samples were measured with LC-MS/MS.

Results: C0, C2, C4-OH, C5, C5-OH, C6, C16, C18 carnitines were lower ( $\mathrm{p}$ value $0.037,0.010,0.005,0.032,0.005$, 0.003, 0.043, 0.003, respectively) and C18:1 carnitine was higher $(\mathrm{p}<0.025)$ in ASD group compared with control group.

Conclusions: Comprehensive carnitine levels for ASD are important to establish a treatment protocol for the treatment of ASD behavior and severity. C18:1 carnitine, detected for the first time in the cases with ASD, is

*Corresponding author: Ozgur Aslan, Department of Clinical Biochemistry Laboratory, Health Sciences University Gazi Yasargil Training and Research Hospital, Uçkuyu Mh., Diyarbakir, Turkey, 21010, E-mail: ozguraslan44@yahoo.com. https://orcid.org/00000003-1890-6268

Burcu Kardaş, Department of Child and Adolescent Psychiatry, Health Sciences University Gazi Yasargil Training and Research Hospital, Diyarbakir, Turkey

Mehmet Nuri Özbek, Department of Child Endocrine, Health Sciences University Gazi Yasargil Training and Research Hospital, Diyarbakir, Turkey

Bahadır Ercan, Department of Biochemistry, Girne American University, Kyrenia, TRNC important for its high levels and for being a glycine transporter two inhibitor. In ASD cases, the molecular analysis might be suggested for enzymes involved in carnitine metabolism and for glycine transporter 2.

Keywords: autism spectrum disorder; C18:1 carnitine; carnitine; dried blood spot samples; liquid chromatography-tandem mass spectrometry.

\section{Introduction}

Autism spectrum disorder (ASD) is a neurodevelopmental disorder with a heterogeneous etiology and a strong genetic basis reveals itself by various clinical symptoms. The main symptoms reveal themselves as deficiencies in social communication and interaction, repetitive behavior patterns, limited interests or activities [1, 2]. Beyond the basic behavioral symptoms in individuals with ASD, gastrointestinal, immune, hepatic and endocrine systems are seen to be affected. Common comorbidities include neurological, psychiatric and physical conditions: neurological comorbidities include epilepsy, sleep disturbance, sensory abnormalities, and motor function delays and/or deficiencies; psychiatric conditions include depression, anxiety, irritability and attention deficit hyperactivity disorder, and physical health problems include chronic gastrointestinal discomfort [3]. The incidence of one or more non-ASD developmental diagnoses is as high as $83 \%$ [4]. It is associated with impaired quality of life and the lifetime cost of supporting an individual with ASD is a very high economic expense due to comorbid disorders $[2,5]$. Estimates of total ASD prevalence ranged from 13.1 to 29.3 per 1,000 children over the age of 8 years. Males have four times more ASD than females [1]. ASD prevalence estimates also vary according to race and ethnicity [6].

Genetic causes of ASD are identified in approximately $30-35 \%$ of cases. For the remaining $65-70 \%$ of cases, there is a general agreement among researchers that ASD may be caused by the interaction of the combination of 
environmental and different genetic factors. Environmental factors can also take part and be reflected in epigenetic modifications [7].

Carnitine (B-hydroxy- $\gamma$-Ntrimethylaminobutyric acid) is necessary for the transfer of long-chain fatty acids along the internal mitochondrial membrane for the continuation of $\beta$-oxidation [8-10]. Carnitine plays a critical role in the energy balance between the cell membranes and in the energy metabolism of tissues that obtain most of their energy from fatty acid oxidation, such as cardiac and skeletal muscles. Although carnitine plays a major role in free fatty acid metabolism, carnitine increases the use of carbohydrates [11]. Carnitine can be synthesized endogenously by the human body, is very low in fruits, vegetables and grains, moderate in dairy products and high in meat, and can also be provided by the food supply. Therefore, genetic disorders in carnitine biosynthesis do not routinely cause low plasma carnitine levels [8-10, 12].

Carnitine deficiency has primary and secondary genetic forms. Secondary deficiency is caused by various fat oxidation defects and organic acidemias that lead to carnitine deficiency due to the loss of urine of acylcarnitines accumulated to enzyme deficiency. Secondary deficiency is caused by various fat oxidation defects and organic acidemias that lead to carnitine deficiency due to the loss of urine of acylcarnitines accumulated to enzyme deficiency. Primary systemic carnitine deficiency is caused by biallelic loss of function mutations in the SLC22A5 gene encoding plasma membrane organic cation transporter 2 (OCTN2) [10, 13, 14]. OCTN2 deficiency is characterized by excessive carnitine loss in the urine leading to systemic deficiency associated with skeletal myopathy, cardiomyopathy, fatty liver and hypoglycemia. Although the possibility of primary systemic carnitine deficiency due to a defect in carnitine biosynthesis has long been presumed a long time ago, primary carnitine biosynthesis disorders have not been described to date $[10,14]$.

Administration of carnitine to patients is the main approach of treatment for systemic carnitine deficiency and is useful in some genetic forms of secondary carnitine deficiency. Administration of carnitine and acetylcarnitine had been investigated for the treatment of many diseases including diabetic peripheral neuropathy, heart failure and mitochondrial disorders, and as an antioxidant [10].

Although autism spectrum disorder has been considered to be highly inherited and caused by many gene interactions, no single gene has been identified that adequately explains the alarmingly increasing prevalence and complex heterogeneity of the disease. As a result of these studies, the relationship between $\mathrm{L}$-carnitine and autism is based on observations such as abnormal mitochondrial function occurring in ASD cases, the relationship between L-carnitine levels and the severity of autism, and genetic aspects of L-carnitine metabolism [15].

Carnitine levels in ASD are important because they show potential mitochondrial dysfunction and abnormal fatty acid metabolism [16]. Although in their studies of Filipek et al. [17], and Mostafa et al. [18], low carnitine levels in ASD children, besides, in their studies of Geier et al. [19], and Fahmy et al. [20], improvement in cases administrated L-carnitine in ASD symptoms have been demonstrated, there are not many studies on this subject. For all these reasons, in this study, in ASD children carnitine levels in dried blood spot samples were evaluated and compared with the control group.

\section{Materials and methods}

The individuals who were diagnosed with ASD (DSM 5) and whose carnitines levels were studied included in the study. The results of the individuals with ASD between 2016 and 2018 were discussed. The control group consisted of the volunteer individuals who did not have any neurological and metabolic findings and have applied to the other clinics of the hospital. The study was approved by the local ethics committee (No: 05.10.2018-144).

A total of 23 children who were diagnosed ASD by Children and Adolescence Clinics of Hospital were included in the study, (19 boys aged between 5 and $18 \mathrm{y} / 0$ and four girls aged between 5 and $16 \mathrm{y} / \mathrm{o}$ ). A total of 24 children were included in this study as a control group, including age-matched 19 boys and five girls. All participants did not have any special diets, and the group with ASD had one Pica disorder. However, the participants were not diagnosed any metabolic disorders.

The samples for fasting carnitine levels in our hospital are taken into dried blood spot cards (Ministry of Health Dried Blood Spot Filter Paper for phenylketonuria screening Lot: 77427W-112, Turkey). All samples were studied with Tandem Gold liquid chromatographytandem mass spectrometry (LC-MS/MS) System (Zivak Technologies, Turkey) and original kits (Ref. no: ZV-3017-0500-15) used in our routine laboratory. Briefly, the test principle is that carnitines are extracted from dry blood samples by organic solvents that includes internal standard. Extracted analytes are derivatized with reagents. Derivatized carnitines are analyzed in LC-MS/MS system. Isotope dilution method is used to calculate the results. The concentration of analytes is calculated according to internal standard concentration fields. A total of 17 carnitine levels including free L-carnitine (FC or C0), acetyl-L-carnitine (ALC or C2); propionyl-L-carnitine (C3); butyryl-L-carnitine (C4); 3-hydroxybutyryl-L-carnitine (C4-OH); isovaleryl-L-carnitine (C5); glutaryl-L-carnitine (C5-DC); 3-hydroxyisovaleryl-L-carnitine (C5-OH); hexanoyl-L-carnitine (C6); adipylL-carnitine (C6-DC); octanoyl-L-carnitine (C8); decanoyl-L-carnitine (C10); dodecanoyl-L-carnitine (C12); myristoyl-L-carnitine (C14); palmitoyl-L-carnitine (C16); stearoyl-L-carnitine (C18); oleoylL-carnitine (C18:1) were measured with the device. 


\section{Statistical analysis}

Statistical analyses were carried out using SPSS (Statistical Package for Social Sciences, Chicago, IL, USA) software. Shapiro-Wilk test was used for normal distribution of data and the Mann-Whitney U test was performed for the difference between groups. $\mathrm{p}<0.05$ was considered statistically significant.

\section{Results}

The mean diagnosis age of children with ASD was 3.00. Comorbidities were Mild (11 children), Moderate
( 9 children) severe ( 2 children) and Profound ( 0 children) intellectual disability, borderline intellectual functioning (BIF) (1 child). There was a child with an EEG defect, there 2 (two) children having seizures (Table 1).

In the ASD group, 18 children were born with normal spontaneous vaginal delivery (NSVD), and five children with caesarean section (C/S); in the normal group, 14 children were born with NSVD and 10 children with C/S. Some of the ASD children have relatives and/or family members with ASD (10 children and two of them were siblings) where there was only one child in the control group. Children with ASD were using or stopped using medications (risperidone,

Table 1: Clinical and family characteristics of children included in the study.

\begin{tabular}{|c|c|c|}
\hline & ASD $(n=23)$ & Control $(n=24)$ \\
\hline \multirow{2}{*}{ Gender } & Female:4 (17.3\%) & Female: 5 (20.8\%) \\
\hline & Male: 19 (82.6\%) & Male: $19(79.1 \%))$ \\
\hline Chronological age, year* & $11.52(\min : 5.10-\max : 18.27)$ & $11.64(\min : 5.43-\max : 18.37)$ \\
\hline Weight, kg* & 47.61 (min:19-max:85) & 41.38 (min:18-max:78) \\
\hline Height, $\mathrm{cm}^{\star}$ & $147.22(\min : 114-\max : 173)$ & 146.17 (min:110-max:176) \\
\hline Age of diagnosis, years & 3.00 (SD: $1.23, \min : 1.20-\max : 5)$ & No \\
\hline \multirow[t]{3}{*}{ Comorbidity } & ADHD: $30.4 \%(n=7)$ & No \\
\hline & Pica: $4.3 \%(n=1)$ & \\
\hline & Stuttering: $4.3 \%(n=1)$ & \\
\hline \multirow[t]{5}{*}{ Accompanying intellectual disability } & Mild:11 & No \\
\hline & Moderate:9 & \\
\hline & Severe:2 & \\
\hline & Profound:0 & \\
\hline & BIF: 1 & \\
\hline \multirow[t]{4}{*}{ Accompanying physical illness } & Visual disabilities: 2 & No \\
\hline & Hearing disability: 2 & \\
\hline & $\mathrm{BMI}>25: 2$ & \\
\hline & No: 17 & \\
\hline \multirow[t]{2}{*}{ Seizure history } & Yes: 2 & No \\
\hline & No: 21 & \\
\hline \multirow[t]{2}{*}{ Drug use } & Yes: 16 & Yes: 2 \\
\hline & No:7 & No: 22 \\
\hline \multirow[t]{3}{*}{ Birth trauma } & Yes: 4 & Yes: 1 \\
\hline & No: 17 & No: 23 \\
\hline & Unknown: 2 & Premature: 1 \\
\hline \multirow[t]{2}{*}{ Birth type } & NSVD: 18 & NSVD: 14 \\
\hline & C/S: 5 & C/S: 10 \\
\hline Age of mother (at birth) & 31.09 (min:22-max: 41) & 31.17 (min:22-max: 38) \\
\hline Age of father (at birth) & 36.57 (min:26-max: 52) & 35.33 (min:27-max: 43) \\
\hline \multirow[t]{2}{*}{ Consanguineous marriage } & Yes: 9 & Yes: 3 \\
\hline & No:14 & No:21 \\
\hline \multirow[t]{2}{*}{ The presence of autism in the family } & Yes:10 & Yes: 1 \\
\hline & No: 13 & No: 23 \\
\hline \multirow[t]{2}{*}{ The presence of psychiatric disease in the family } & Yes: 14 & Yes: 4 \\
\hline & No: 9 & No: 20 \\
\hline \multirow[t]{2}{*}{ Smoking in the mother during pregnancy } & Yes: 10 & Yes: 2 \\
\hline & No: 13 & No: 22 \\
\hline \multirow[t]{2}{*}{ Smoking in the father } & Yes: 11 & Yes: 16 \\
\hline & No: 12 & No: 8 \\
\hline
\end{tabular}

${ }^{*} \mathrm{p}$ value 0,05 Mann-Whitney U test. BIF, Borderline Intellectual Functioning; BMI, Body mass index; NSVD, Normal spontaneous vaginal delivery; $\mathrm{C} / \mathrm{S}$, Cesarean section. 
Table 2: Dried blood spot carnitine levels in both groups.

\begin{tabular}{|c|c|c|c|c|c|c|}
\hline Carnitine & Grup & $\mathbf{N}$ & Mean, $\mu \mathrm{mol} / \mathrm{L}$ & SD & Reference ranges, $\mu \mathrm{mol} / \mathrm{L}$ & Limits of detection ( $\mu \mathrm{mol} / \mathrm{L})$ \\
\hline \multirow[t]{2}{*}{$\mathrm{CO}^{*}$} & ASD & 23 & 35.699 & 9.299 & $12-220$ & 0.023 \\
\hline & Control & 24 & 41.594 & 9.215 & & \\
\hline \multirow[t]{2}{*}{$\mathrm{C} 2 *$} & ASD & 23 & 16.365 & 3.627 & $5-85$ & 0.042 \\
\hline & Control & 24 & 19.410 & 3.913 & & \\
\hline \multirow[t]{2}{*}{$\mathrm{C} 3$} & ASD & 23 & 2.086 & 0.630 & $0-6.5$ & 0.024 \\
\hline & Control & 24 & 2.441 & 0.808 & & \\
\hline \multirow[t]{2}{*}{$\mathrm{C} 4$} & ASD & 23 & 0.276 & 0.119 & $0-2$ & 0.054 \\
\hline & Control & 24 & 0.267 & 0.072 & & \\
\hline \multirow[t]{2}{*}{$\mathrm{C} 4-\mathrm{OH}^{*}$} & ASD & 23 & 0.149 & 0.060 & $0-0.5$ & 0.047 \\
\hline & Control & 24 & 0.175 & 0.030 & & \\
\hline \multirow[t]{2}{*}{$\mathrm{C} 5^{*}$} & ASD & 23 & 0.159 & 0.047 & $0-1.49$ & 0.023 \\
\hline & Control & 24 & 0.191 & 0.054 & & \\
\hline \multirow[t]{2}{*}{$\mathrm{C} 5-\mathrm{OH}^{\star}$} & ASD & 23 & 0.316 & 0.130 & $0-1.2$ & 0.022 \\
\hline & Control & 24 & 0.417 & 0.139 & & \\
\hline \multirow[t]{2}{*}{$C 5-D C$} & ASD & 23 & 0.194 & 0.060 & $0-0.35$ & 0.076 \\
\hline & Control & 24 & 0.217 & 0.054 & & \\
\hline \multirow[t]{2}{*}{ C6* } & ASD & 23 & 0.092 & 0.028 & $0-0.7$ & 0.041 \\
\hline & Control & 24 & 0.114 & 0.020 & & \\
\hline \multirow[t]{2}{*}{ C6-DC } & ASD & 23 & 0.087 & 0.041 & $0-0.5$ & 0.024 \\
\hline & Control & 24 & 0.087 & 0.031 & & \\
\hline \multirow[t]{2}{*}{$\mathrm{C} 8$} & ASD & 23 & 0.140 & 0.038 & $0-0.9$ & 0.088 \\
\hline & Control & 24 & 0.130 & 0.030 & & \\
\hline \multirow[t]{2}{*}{ C10 } & ASD & 23 & 0.177 & 0.064 & $0-0.7$ & 0.088 \\
\hline & Control & 24 & 0.161 & 0.044 & & \\
\hline \multirow[t]{2}{*}{$\mathrm{C} 12$} & ASD & 23 & 0.140 & 0.043 & $0-1.44$ & 0.068 \\
\hline & Control & 24 & 0.141 & 0.030 & & \\
\hline \multirow[t]{2}{*}{ C14 } & ASD & 23 & 0.139 & 0.036 & $0-1.1$ & 0.067 \\
\hline & Control & 24 & 0.130 & 0.033 & & \\
\hline \multirow[t]{2}{*}{ C16* } & ASD & 23 & 1.123 & 0.277 & $0-2.1$ & 0.077 \\
\hline & Control & 24 & 1.380 & 0.437 & & \\
\hline \multirow[t]{2}{*}{ C18* } & ASD & 23 & 0.839 & 0.213 & $0-7$ & 0.042 \\
\hline & Control & 24 & 1.230 & 0.470 & & \\
\hline \multirow[t]{2}{*}{ C18:1* } & ASD & 23 & 0.547 & 0.115 & $0-4$ & 0.091 \\
\hline & Control & 24 & 0.478 & 0.151 & & \\
\hline
\end{tabular}

SD, Standard deviation. *p value $<0.05$ significant.

atomoxetine, haloperidol, naltrexone, aripiprazole) for major symptoms such as behavior problems, aggression, offensiveness. In the control group, one child was using medication for anxiety disorder and one child for asthma (fluoxetine, hydroxyzine, salbutamol, montelukast and desloratadine) (Table 1).

Familial characteristics of the children were; The maternal age (at birth) was 31 (min: 22-max: 41) in the ASD group and 31 (min: 22-max: 38) in the control group; mean age of fathers (at birth) was 36(min: 26-max: 52) in the ASD group and 35 (min: 27-max: 43) in the control group; The consanguineous marriage was nine in the ASD group and three in a control group; The presence of autism in the family was 10 in the ASD group and one in the control group; The prevalence of the psychiatric disease in families was 14 in the ASD group and four in the control group; Smoking of the mother during pregnancy was 10 in the ASD group and two in the control group; Smoking of the father was 11 in the ASD group and 16 in the control group (Table 1).

Carnitine levels are shown as mean, standard deviation and minimum-maximum in Table 2.

In the two ASD cases for C8 carnitine, one control $(0.88 \mu \mathrm{mol} / \mathrm{L})$, two ASD cases for C10 carnitine, one control $(0.88 \mu \mathrm{mol} / \mathrm{L})$, one ASD cases for $\mathrm{C} 12$ carnitine $(0.68 \mu \mathrm{mol} /$ $\mathrm{L}$ ), the values were below the limit of detection, the lower limit of detection was taken into account while performing statistical analyses.

In the ASD group, a significant decrease in $\mathrm{C} 0, \mathrm{C} 2$, C4-OH, C5, C5-OH, C6, C16, C18 carnitines compared to the control group (respectively, $\mathrm{p}$ value $0.037,0.010$, $0.005,0.032,0.005,0.003,0.043,0.003$ ), and a significant increase in C18: 1 carnitine $(\mathrm{p}<0.025)$ was observed in the mean dried blood spot samples (Table 2) (Figure 1). 

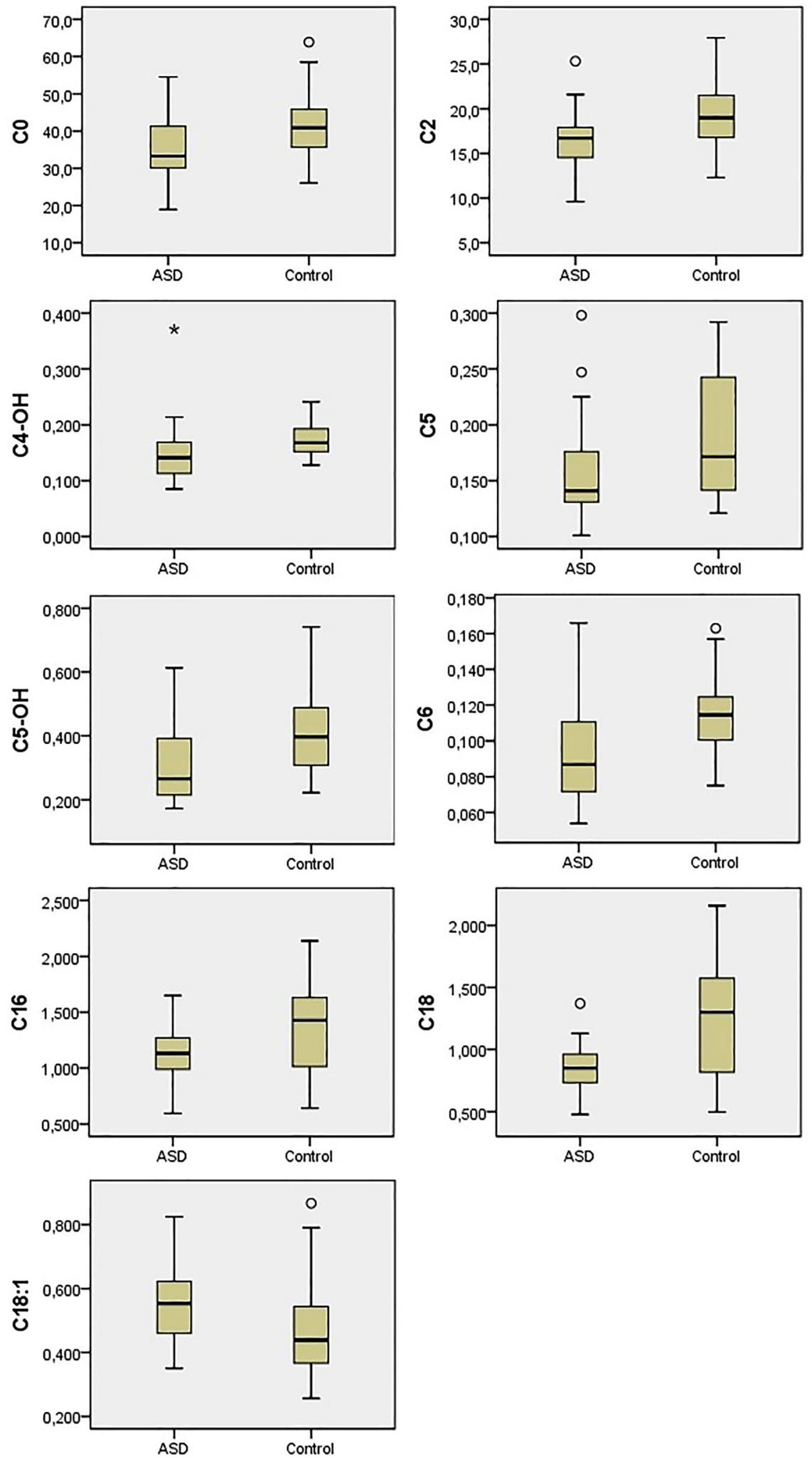

Figure 1: Carnitines with statistical significant difference between ASD and control group. 


\section{Discussion}

Carnitine levels were analyzed with dried blood spot samples by LC-MS/MS method in 23 ASD cases. According to the control group, carnitines of $\mathrm{C} 0, \mathrm{C} 2, \mathrm{C} 4-\mathrm{OH}, \mathrm{C}$, $\mathrm{C} 5-\mathrm{OH}, \mathrm{C} 6, \mathrm{C} 16$ and $\mathrm{C} 18$ are low and carnitine of C18: 1 is high, other carnitine levels were similar.

In their meta-analysis of Rossignol and Frye [21], it has been reported that the prevalence of mitochondrial disorders (5\%) was significantly higher in cases with ASD than in the general population and that ASD was associated with mitochondrial disorders. The prevalence of the biomarker values of mitochondrial dysfunction with ASD was much higher than the prevalence of mitochondrial disorders. It has been reported that the values of many mitochondrial biomarkers (lactate, pyruvate, carnitine, and ubiquinone) differ significantly between ASD and controls, and some markers are associated with the severity of ASD [21]. Carnitine is thought to exert protective effects on mitochondria and all cells by inhibiting free fatty acid-induced mitochondrial membrane damage and/or secondary effects [22, 23].

In our study, it has been reported that for certain symptoms drugs were administered by some of the ASD children or some of them stopped the treatment, in one of the cases cortexin (contains polypeptides, vitamins, minerals and amino acids) was administered but was quit for a long time ago, in two cases probiotics and dietary supplements (fish oil and B vitamins) were received. In our study, there were no cases using carnitine. Among ASD cases with mitochondrial disorders, the use of carnitine is highlighted as one of the most recommended nutritional supplements [21, 24, 25].

In the study of Geier et al. [19], it has been reported that there is a significant improvement in several clinical measurements of ASD severity, that is in 30 cases diagnosed with ASD and randomly assigned as liquid L-carnitine $(n=19)$ and placebo ( $\mathrm{n}=11$ ) for 3 months. Similarly, Fahmy et al. [18] have been reported that 30 cases diagnosed with autism were randomly assigned to receive of liquid l-carnitine $(\mathrm{n}=16)$ or placebo ( $\mathrm{n}=14)$ for 6 months, and after 6 months, liquid 1-carnitine therapy administered significantly improved the autistic behavior and decreased the severity of autism. Total and free carnitine levels were examined in the studies [19, 20]. Based on the data obtained from these studies and our study, it is obvious that carnitine levels are important for ASD and it should be emphasized that carnitine levels should be examined in terms of ASD treatment protocols.
In the study of Lv et al. [16], it has been reported that levels of free carnitine (C0), glutaryl carnitine (C5-DC), octyl carnitine, carnosyl carnitine (C26) were found to be significantly lower in 60 children ( 49 boys and 11 girls) diagnosed with ASD (42.86 \pm 11.03 months) and 30 children (25 boys and five girls) with typically developing (39.32 \pm 12.88 months). As a conclusion, it has been reported that glutaryl carnitine and carnosyl carnitine might be potential biomarkers for diagnosis of ASD, that the limitations of the study were the relatively small sample size and participants which were the children within 6 years of age [16]. In contrast to this aforementioned study, in our study, there is not any significant difference in C0, C5-DC levels compared to the control group and octyl, C26 carnitine levels were not examined. In our study, the age of the ASD group reached up to 18.27 years and it has been stated that in the ASD group compared to the control group $\mathrm{C0}, \mathrm{C} 2, \mathrm{C} 4-\mathrm{OH}, \mathrm{C} 5, \mathrm{C}-\mathrm{OH}, \mathrm{C} 6, \mathrm{C16}$, C18 levels were significantly low, C18:1 level significantly high.

Adams et al. [26] demonstrated that carnitine results in 67 cases with ASD in both groups with and without carnitine administration started at levels similar to neurotypical controls, and that treatment led to a significant increase in L-carnitine and non-significant increase in acetylL-carnitine. In this study, in cases with ASD, it has been stated that administered L-carnitine can be absorbed better than acetyl-L-carnitine in other studies $[19,20]$ and therefore might be more effective. They also reported that the benefit from carnitine was very limited in cases with ASD [26]. In this study [26] carnitine levels were initially similar to the control group so that it is considered to be the reason for the limitation in benefit from carnitine in cases with ASD. Although no protocol for treatment was established in our study, it could be said that carnitine supplementation would be beneficial in cases with ASD with low carnitine levels considering the studies in the literature [19, 20, 26]. But carnitine treatment might be harmful in cases of long-chain fatty acid oxidation disorders (FAODs), as it increases the concentrations of long-chain acylcarnitines, which are potentially arrhythmogenic [27]. Therefore, in carnitine supplementation, long-chain fatty acid oxidation disorders and side effects of carnitine should be taken into consideration.

In our study, it has been proved that only carnitine C18: 1 in the ASD group was statistically significant $(p<0.025)$. Height C18:1 carnitine levels were also determined in carnitine palmitoyltransferase-2 [28], carnitineacylcarnitine translocase [29], long-chain acyl-CoA dehydrogenase deficiencies [30]. 
Clark-Taylor and Clark-Taylor have been stated that C10:1, C14:2 and C14:1 for plasma acyl-carnitine profile were high and that long-chain acyl-CoA dehydrogenase deficiency might be a cause of autism, and it might be important to identify acyl-carnitine profiles in other children with autism [31]. We found that a high level of C18:1 suggests that the studies with long-chain acyl-CoA dehydrogenase and enzymes related to carnitine metabolism should be performed in ASD cases.

Also, carnitine $\mathrm{C} 18: 1$ has a role as a glycine transporter 2 (GlyT2) inhibitor [32, 33]. The main role of GlyT2 is to recapture glycine released in the synaptic cleft and maintain high glycine concentration in the presynaptic neuron $[34,35]$. It is considered that C18:1 carnitine might be interested in symptoms of ASD through GlyT2. Although there is a study on glycine transporter one in cases with ASD [36], no studies on GlyT2 have been found in the literature. GlyT2 inhibitors show potential as analgesics [33] and oleoyl-L-carnitine may have the potential for new research into pain sensation in ASD individuals.

The wideness of age distribution of the cases in this study is important. One of the limitations of our study is the relatively inadequate number of cases. Besides, no treatment protocol was applied in the study. Therefore, the efficacy of carnitine treatment could not be monitored. It is another limitation that the uncertainty of relationships of carnitine C18:1 with long-chain fatty acid oxidation or enzyme disorders in carnitine metabolism is not clarified as urine organic acids were not studied. In addition, this study was conducted on dry blood samples can be stated as a limitation. It migt be confirmed by studies with carnitines to be measured in direct blood samples. Despite all these, it is thought that this study will contribute to the literature considering the small number of studies on carnitine levels in ASD cases.

Comprehensive carnitine levels for ASD are important for the diagnosis of ASD and the treatment in the elimination of ASD behaviors and severity. It is important to determine the relationship between ASD behavior and the severity of educational interventions and carnitine levels in cases with ASD. In our study, although the number of patients was relatively low in cases with ASD, a molecular level analysis could be recommended for enzymes playing a role in carnitine metabolism related to $\mathrm{C} 18: 1$ high levels of carnitine and glycine transporter 2 .

Research funding: This research has not received a specific grant from funding organizations in the public, commercial, or non-profit sectors.
Author contributions: All authors have accepted responsibility for the entire content of this manuscript and approved its submission.

Competing interests: Authors state no conflict of interest.

\section{References}

1. Baio J, Wiggins L, Christensen DL, Maenner MJ, Daniels J, Warren $Z$, et al. Prevalence of autism spectrum disorder among children aged 8 Years - autism and developmental disabilities monitoring network, 11 sites, United States, 2014. MMWR Surveill Summ 2018;67:1-23.

2. Howsmon DP, Kruger U, Melnyk S, James SJ, Hahn J. Classification and adaptive behavior prediction of children with autism spectrum disorder based upon multivariate data analysis of markers of oxidative stress and DNA methylation. PLoS Comput Biol 2017;13: e1005385.

3. Cheng N, Rho JM, Masino SA. Metabolic dysfunction underlying autism spectrum disorder and potential treatment approaches. Front Mol Neurosci 2017;10:34.

4. Levy SE, Giarelli E, Lee LC, Schieve LA, Kirby RS, Cunniff C, et al. Autism spectrum disorder and co-occurring developmental, psychiatric, and medical conditions among children in multiple populations of the United States. J Dev Behav Pediatr 2010;31: 267-75.

5. De Rubeis S, Buxbaum JD. Genetics and genomics of autism spectrum disorder: embracing complexity. Hum Mol Genet 2015; 24:R24-31.

6. Jo H, Schieve LA, Rice CE, Yeargin-Allsopp M, Tian LH, Blumberg SJ, et al. Age at autism spectrum disorder (ASD) diagnosis by race, ethnicity, and primary household language among children with special health care needs, United States, 2009-2010. Matern Child Health J 2015;19:1687-97.

7. Anwar A, Abruzzo PM, Pasha S, Rajpoot K, Bolotta A, Ghezzo A, et al. Advanced glycation endproducts, dityrosine and arginine transporter dysfunction in autism - a source of biomarkers for clinical diagnosis. Mol Autism 2018;9:1-16.

8. Longo N. Primary carnitine deficiency and newborn screening for disorders of the carnitine cycle. Ann Nutr Metab 2016;68:5-9.

9. Longo N, Frigeni M, Pasquali M. Carnitine transport and fatty acid oxidation. Biochim Biophys Acta 2016;1863:2422-35.

10. Celestino-Soper PB, Violante S, Crawford EL, Luo R, Lionel AC, Delaby $E$, et al. A common X-linked inborn error of carnitine biosynthesis may be a risk factor for nondysmorphic autism. Proc Natl Acad Sci U S A 2012;109:7974-81.

11. Flanagan JL, Simmons PA, Vehige J, Willcox MD, Garrett Q. Role of carnitine in disease. Nutr Metab 2010;7:30.

12. Malaguarnera $M$, Cauli 0 . Effects of I-carnitine in patients with autism spectrum disorders: review of clinical studies. Molecules 2019;24:4262.

13. El-Hattab AW, Scaglia F. Disorders of carnitine biosynthesis and transport. Mol Genet Metabol 2015;116:107-12.

14. Longo N, Amat di San Filippo C, Pasquali M. Disorders of carnitine transport and the carnitine cycle. Am J Med Genet C Semin Med Genet 2006;142C:77-85. 
15. Demarquoy C, Demarquoy J. Autism and carnitine: a possible link. World J Biol Chem 2019;10:7-16.

16. Lv QQ, You C, Zou XB, Deng HZ. Acyl-carnitine, C5DC, and C26 as potential biomarkers for diagnosis of autism spectrum disorder in children. Psychiatr Res 2018;267:277-80.

17. Filipek PA, Juranek J, Nguyen MT, Cummings C, Gargus JJ. Relative carnitine deficiency in autism. J Autism Dev Disord 2004;34:615-23.

18. Mostafa GA, El-Gamal HA, El-Wakkad AS, El-Shorbagy OE, Hamza MM. Polyunsaturated fatty acids, carnitine and lactate as biological markers of brain energy in autistic children. Int J Child Neuropsychiatry 2005;2:179-88.

19. Geier DA, Kern JK, Davis G, King PG, Adams JB, Young JL, et al. A prospective double-blind, randomized clinical trial of levocarnitine to treat autism spectrum disorders. Med Sci Mon Int Med J Exp Clin Res 2011;17:PI15-23.

20. Fahmy SF, El-hamamsy MH, Zaki OK, Badary OA. I-Carnitine supplementation improves the behavioral symptoms in autistic children. Res Autism Spectr Disord 2013;7:159-66.

21. Rossignol DA, Frye RE. Mitochondrial dysfunction in autism spectrum disorders: a systematic review and meta-analysis. Mol Psychiatr 2012;17:290-314.

22. Chang B, Nishikawa M, Nishiguchi S, Inoue M. L-carnitine inhibits hepatocarcinogenesis via protection of mitochondria. Int J Canc 2005;113:719-29.

23. Sharma S, Black SM. Carnitine homeostasis, mitochondrial function, and cardiovascular disease. Drug Discov Today Dis Mech 2009;6:e31-39.

24. Delhey LM, Nur Kilinc E, Yin L, Slattery JC, Tippett ML, Rose S, et al. The effect of mitochondrial supplements on mitochondrial activity in children with autism spectrum disorder. J Clin Med 2017;6:18.

25. Gargus JJ, Imtiaz F. Mitochondrial energy-deficient endophenotype in autism. Am J Biochem Biotechnol 2008;4: 198-207.

26. Adams JB, Audhya T, Geis E, Gehn E, Fimbres V, Pollard EL, et al. Comprehensive nutritional and dietary intervention for autism spectrum disorder-a randomized, controlled 12-month trial. Nutrients 2018;10:369.
27. Stanley CA, Bennet MJ, Mayatepek E. Disorders of mitochondrial fatty acid oxidation and related metabolic pathways. In: Saudubray J-M, van den Berghe G, Walter JH, editors Inborn Metabolic Diseases: Diagnosis and Treatment, 5 th ed. Berlin Heidelberg: Springer-Verlag; 2012. p. 176-87pp.

28. Gempel K, Kiechl S, Hofmann S, Lochmüller H, KiechlKohlendorfer U, Willeit J, et al. Screening for carnitine palmitoyltransferase II deficiency by tandem mass spectrometry. J Inherit Metab Dis 2002;25:17-27.

29. Yan HM, Hu H, Ahmed A, Feng BB, Liu J, Jia ZJ, et al. Carnitineacylcarnitine translocase deficiency with c.199-10 T>G and novel c. $1 \mathrm{~A}>\mathrm{G}$ mutation: two case reports and brief literature review. Medicine 2017;96: e8549.

30. Spiekerkoetter U, Tokunaga C, Wendel U, Mayatepek E, Exil V, Duran $M$, et al. Changes in blood carnitine and acylcarnitine profiles of very long-chain acyl-CoA dehydrogenase-deficient mice subjected to stress. Eur J Clin Invest 2004;34:191-6.

31. Clark-Taylor T, Clark-Taylor BE. Is autism a disorder of fatty acid metabolism? Possible dysfunction of mitochondrial betaoxidation by long chain acyl-CoA dehydrogenase. Med Hypotheses 2004;62:970-5.

32. PubChem. Oleoyl-L-carnitine. Available from: https://pubchem. ncbi.nlm.nih.gov/compound/Oleoyl-L-carnitine. [Accessed 19 July 2019].

33. Carland JE, Mansfield RE, Ryan RM, Vandenberg RJ. OleoylL-carnitine inhibits glycine transport by GlyT2. Br J Pharmacol 2013;168:891-902.

34. Morrow JA, Collie IT, Dunbar DR, Walker GB, Shahid M, Hill DR. Molecular cloning and functional expression of the human glycine transporter GlyT2 and chromosomal localisation of the gene in the human genome. FEBS Lett 1998;439:334-40.

35. Zafra F, Ibáñez I, Giménez C. Glycinergic transmission: glycine transporter GlyT2 in neuronal pathologies. Neuronal Signal 2016; 1: NS20160009.

36. Yang P, Lane HY, Yen CF, Chang CL. A pilot open-label trial of use of the Glycine transporter I inhibitor, sarcosine, in highfunctioning children with autistic disorder. Transl Med 2014;04. https://doi.org/10.4172/2161-1025.1000127. 